\title{
Relações entre a saúde mental de estudantes do ensino fundamental e as práticas e estilos parentais"
}

\author{
The relationships between the mental health of elementary school students \\ and the practices and parenting styles
}

504

\begin{abstract}
Resumo
O presente estudo teve por objetivo, relacionar a saúde mental infantil de crianças estudantes do primeiro ciclo do ensino fundamental com as práticas e estilos parentais dos responsáveis. Foram participantes da pesquisa 321 crianças, estudantes do primeiro ao quinto ano do ensino fundamental e seus responsáveis. Os instrumentos de medida utilizados foram: o Questionário de Capacidades e Dificuldades, o Inventário de Estilos Parentais e o Questionário de Atividades Cotidianas. Foram feitas análises estatísticas comparativas e correlacionais. Os resultados indicaram correlações positivas significativas entre saúde mental infantil e as práticas e estilos parentais. O estudo também revelou que nas famílias que adotam regras e responsabilidades que são compartilhadas por todos, as crianças se comportam mais de forma pró-social, apontando um possível fator de proteção à saúde mental infantil. O estudo também revelou que nas famílias que adotam regras e responsabilidades que são compartilhadas por todos, as crianças se comportam mais de forma pró-social, apontando um possível fator de proteção à saúde mental infantil.
\end{abstract}

Palavras-chave: Saúde Mental Infantil. Estilos Parentais. Práticas Parentais. Ensino Fundamental.

\begin{abstract}
Objectives: This study aimed to relate to children's mental health of school children of the first cycle of basic education with parenting practices and styles of those responsible. Methods: This study included a total of 321 first- to fifth-grade children and their parents or guardians. The measurement instruments used for data collection were the strengths and difficulties questionnaire, the parenting styles inventory and the activities of daily life questionnaire. Correlation analyses and comparative analyses were performed. Results: The results indicated significant correlations between parenting practices and child mental health. This study also suggested that in families where boundaries, rules and roles were established, including shared responsibilities, they have been shown to be associated with higher rates of child pro-social behavior, indicating protective factors for the mental health outcomes of children. Conclusions: The study revealed that there are links between child mental health, and the parenting practices and styles adopted by those responsible in order that the more positive practices, the fewer difficulties in mental health children present. It is believed that the results may contribute towards subsidizing relevant issues to be considered by public policies of child mental health.
\end{abstract}

Keywords: Childhood's Mental Health. Parenting Styles. Parenting Practices. Basic Education.

DOI: $10.15343 / 0104-7809.20153904504513$

\# O presente manuscrito é parte da Tese de Doutorado da primeira autora, defendida junto ao Programa de Pós-Graduação em Educação Especial da Universidade Federal de São Carlos.

* Universidade Federal de São Carlos - SP - Brasil. E-mail: mariafernandacid@gmail.com

** Universidade Federal de São Carlos - SP - Brasil. E-mail: thelmamatsukura@gmail.com

*** Universidade Federal de São Carlos - SP - Brasil. E-mail: fabianacia@hotmail.com

Os autores declaram não haver conflitos de interesse. 


\section{INTRODUÇÃO}

A identificação de problemas relativos à saúde mental infantil e dos processos que envolvem sua ocorrência é fundamental para os campos da saúde e educação, na medida em que permitem o desenvolvimento de políticas de intervenção direcionadas à prevenção e/ou promoção da saúde mental de crianças ${ }^{1,2}$. A saúde mental infantil pode ser compreendida enquanto um elenco de habilidades adaptativas que envolvem aspectos emocionais, comportamentais e sociais. Os aspectos presentes no ambiente (como por exemplo, práticas parentais inadequadas, baixa estimulação infantil) onde a criança vive são os mais envolvidos na determinação da saúde mental infantil ${ }^{3,4,5}$.

Sobre a identificação de problemas relativos à saúde mental na população de crianças e adolescentes, o estudo de Sá e colaboradores indicou que, a partir do ano 2000, estudos epidemiológicos na área de saúde mental da infância e adolescência começaram a ser realizados em países em desenvolvimento, incluindo o Brasil. As taxas encontradas são semelhantes às de países desenvolvidos, cujas estimativas atuais sinalizam que uma entre quatro ou cinco crianças no mundo sofre algum transtorno mental ${ }^{2}$.

Outros estudos buscaram investigar aspectos relacionados à saúde mental infantil, tanto no que se refere às questões epidemiológicas, quanto em relação aos fatores que podem ou não estar relacionados à presença e/ou ausência de problemas emocionais em crianças. Por meio destes estudos foi possível identificar alguns fatores de risco e de e de proteção, tais como nível socioeconômico, saúde mental materna, escolaridade dos pais, recursos presentes no ambiente familiar, tais como brinquedos e material escolar, que estão presentes nas esferas individual, familiar e extrafamiliar ${ }^{3,6,7}$.

Paula et al., ${ }^{8}$ fizeram uma revisão de estudos epidemiológicos de cunho populacional realizados no Brasil de 1980 a 2009. Os autores encontraram 15 estudos publicados no período de 2001 a 2009 que apontaram taxas de prevalência de saúde mental variando de $12 \%$ a $24,6 \%$ com base em instrumentos de rastreamento e de $7 \%$ a 12,7\% com base em instrumentos de diagnóstico. Verificou-se uma discrepância regional dos estudos, na medida em que todas as pesquisas desenvolvidas foram realizadas em apenas três estados (São Paulo, Porto Alegre e Bahia) ${ }^{8}$.

Além da questão epidemiológica, pesquisas mais recentes têm buscado investigar de forma mais veemente os fatores relacionados à manifestação ou não dos problemas de saúde mental nessa população, ou seja, os fatores de risco e proteção. No entanto, o principal enfoque tem sido dado aos fatores de risco, em especial, àqueles relacionados ao contexto e ambiente familiar $2,3,7,8$.

Nessa direção, alguns estudos nacionais buscaram identificar fatores de risco relacionados ao desenvolvimento de problemas de saúde mental infantil. Ferrioli et al. ${ }^{7}$ desenvolveram um estudo que objetivou identificar crianças em risco para transtornos emocionais nos usuários de um Núcleo de Atenção Primária do Programa de Atenção Primária e Saúde da Família. Participaram 100 crianças de seis a 12 anos de idade. Os autores encontraram associações entre a presença de transtornos emocionais nas crianças e a presença de situações adversas nos contextos familiar e escolar (tais como estresse materno, instabilidade financeira da família e ausência de rotinas definidas) e também de dificuldades escolares ${ }^{7}$.

Sobre as práticas e estilos parentais, no dia a dia familiar, os pais buscam guiar o comportamento dos filhos a fim de que os mesmos sigam princípios morais e aprendam comportamentos que garantam a independência, a autonomia e responsabilidade. Assim, os filhos podem exercer adequadamente o papel social quando jovens e adultos ${ }^{9,10,11 .}$

Para isso, os pais utilizam diversas estratégias e técnicas para orientar o comportamento dos filhos no cotidiano e promover sua independência e socialização. Tais estratégias e técnicas utilizadas pelos pais na educação dos filhos são denominadas práticas educativas parentais ${ }^{10,11,12}$.

O resultado do uso de um conjunto de Práticas Educativas Parentais é denominado Estilo Parental ${ }^{10,11,13}$, que pode ser entendido como a 
maneira que os pais lidam com as questões de poder, hierarquia e apoio emocional na relação com os filhos. Já o Estilo Parental é o padrão global de características da interação dos pais com os filhos em diversas situações, gerando um clima emocional ${ }^{12}$.

Com o objetivo de investigar crianças com risco para o desenvolvimento de problemas de conduta, bem como a influência das variáveis práticas parentais e uso do tempo livre no desenvolvimento de tais problemas, Yu et al. ${ }^{14}$ avaliaram 4936 crianças australianas de quatro e cinco anos de idade. Os resultados encontrados revelaram que as principais variáveis relacionadas aos problemas de conduta nas crianças foram a utilização, pelos responsáveis, de práticas parentais pouco consistentes e hostis na educação das crianças. As autoras discutem esse resultado, apontando que práticas parentais dessa natureza podem gerar estresse e medo nas crianças, interferindo na regulação do humor das mesmas e as levando a apresentarem reações imaturas e impulsivas ${ }^{14}$.

Salvo et al., ${ }^{13}$ desenvolveram um estudo com o objetivo de levantar quais práticas parentais avaliadas pelo Inventário de Estilos Parentais de Gomide ${ }^{10}$ poderiam ser preditoras de problemas de comportamento identificados pelo Child Behavior Check List (CBCL). Foram participantes, 30 crianças, com idades entre 11 e 13 anos, da rede pública de ensino, e um de seus pais. Os resultados revelaram que as práticas parentais monitoria positiva e comportamento moral são variáveis preditoras de comportamentos pró-sociais, e sua falta somada às práticas negativas são preditoras de dificuldades comportamentais ${ }^{13}$.

Assim, observa-se que embora estudos internacionais e nacionais já indiquem a importância das práticas e estilos parentais na determinação da saúde mental infantil, existe a necessidade de mais investigações que busquem compreender de forma mais aprofundada essa questão, no que diz respeito a especificar as práticas e os comportamentos que derivam das mesmas, a partir de outras características também presentes na relação pai/mãe-criança e no ambiente familiar tais como as relações de cuidado existentes na família. Acredita-se que tais estudos possam contribuir de forma mais efetiva com o planejamento e implementação de intervenções preventivas em saúde e educação, visando a promoção da saúde mental infantil.

Com base no exposto, o presente estudo teve por objetivo, relacionar a saúde mental infantil de crianças estudantes do primeiro ciclo do ensino fundamental com as práticas e estilos parentais dos responsáveis.

\section{MÉTODOS}

Foram participantes do presente estudo 321 responsáveis por crianças estudantes do primeiro ao quinto ano do ensino fundamental de cinco escolas da rede municipal de ensino da cidade de São Carlos, interior do Estado de São Paulo. Em relação às crianças, 49\% delas são do gênero masculino e $51 \%$ do gênero feminino. $39 \%$ delas possuem de seis a oito anos, $43 \%$ de nove a dez anos e $18 \%$ acima de dez anos. Do total de responsáveis, $84 \%$ eram as mães das crianças, $7 \%$ os pais, $5 \%$ os avós e $3 \%$ outros. $70 \%$ dos responsáveis tinham idade variando de 26 a 45 anos. Em relação à escolaridade, $5 \%$ dos responsáveis eram analfabetos, 32\% tinham ensino fundamental incompleto ou completo, 30\% tinham ensino médio incompleto ou completo. Quanto à renda mensal, 71\% dos participantes declararam renda familiar de até dois salários mínimos.

Os instrumentos utilizados na coleta de dados foram:

\section{Questionário de Atividades Cotidianas -} QAC: Para a identificação dos dados gerais da família e de algumas práticas parentais de cuidado dos responsáveis em relação à criança, tais como: existência de responsabilidades, regras e limites no ambiente familiar, práticas de carinho, diálogo e brincadeiras em conjunto no cotidiano, foi construído pelas pesquisadoras, um questionário com 42 questões abertas e fechadas, apresentado aos responsáveis participantes sob a forma de entrevista. Aponta-se que o questionário foi elaborado a partir de apontamentos da literatura referente aos fatores de risco e proteção que possivelmente estão envolvidos na determinação da saúde mental 
infantil $6,7,15,16$. Não foram realizados estudos de validação deste instrumento.

Questionário de Capacidades e Dificuldades (SDQ): O SDQ ${ }^{17}$ foi utilizado para avaliar problemas de saúde mental infantil. É composto por 25 itens, subdivididos em cinco subescalas que avaliam: hiperatividade, sintomas emocionais, problemas de conduta, relações interpessoais e comportamento pró-social. No presente estudo foi utilizada a versão para pais, que foi apresentada aos responsáveis pelas crianças. Esse questionário tem sido traduzido e validado para mais de 40 países, apresentando tradução, também, para o português ${ }^{6,18}$. Para a amostra do presente estudo foi calculado o Alpha de Cronbach para o SDQ, revelando coeficiente de consistência interna de 0,72.

Inventário de Estilos Parentais (IEP): Para a avaliação dos estilos parentais dos responsáveis participantes foi utilizado o IEP, que foi elaborado a fim de identificar famílias em que haja alta probabilidade de desenvolvimento de comportamentos antissociais em pelo menos um de seus membros ${ }^{10}$. O inventário foi desenvolvido a partir das práticas educativas: monitoria positiva, comportamento moral, negligência, abuso físico, abuso psicológico, disciplina relaxada, punição inconsistente e monitoria negativa. Contém 42 questões e pode ser respondido tanto pelos pais (que respondem sobre como utilizam as práticas educativas com seus filhos), quanto pelos filhos (que respondem sobre as práticas educativas do pai e da mãe separadamente). No presente estudo, utilizou-se apenas a versão para os pais, que foi respondida pelo responsável.

Ressalta-se que esse instrumento apresenta estudos de validação no Brasil $^{20}$, sendo que os resultados para avaliar o estilo parental materno revelaram coeficientes razoáveis de consistência interna, variando de 0,47 (monitoria negativa) a 0,82 (abuso físico). Para a amostra do presente estudo foi calculado o Alpha de Cronbach para o índice de estilo parental (iep), revelando coeficiente de consistência interna de 0,71. Os escores do IEP revelam o Estilo Parental adotado pelos Pais (pai e mãe separadamente) que pode ser: 1. Estilo parental ótimo: com presença importante das práticas parentais positivas e ausência das práticas negativas; Estilo parental regular acima da média trata-se de um estilo bom, mas a autora aconselha a busca de um aprimoramento das práticas parentais; Estilo parental regular abaixo da média, indica que seria importante os pais participarem de grupos de treinamento de pais; Estilo parental de risco, indica que os filhos estão em risco importante para o desenvolvimento de comportamentos anti-sociais. A autora aconselha a participação em programas de intervenção terapêutica para pais, por exemplo, nas situações de abuso físico, abuso psicológico e negligência ${ }^{19}$.

Após a aprovação do projeto pelo Comitê de Ética em Pesquisas em Seres Humanos da Universidade Federal de São Carlos (CAAE: 4677.0.000.135-08) e autorização da Secretaria Educação do município de São Carlos, iniciaram-se os procedimentos de identificação e localização dos participantes. A partir do número de crianças matriculadas nas sete escolas municipais que aceitaram participar do estudo (são oito as escolas municipais, mas uma não aceitou participar), através do número total de alunos por escola e por série, foram realizados os procedimentos para identificação da quantidade de participantes necessários para o desenvolvimento do estudo e para a composição da amostra probabilística. Definida a amostra, um novo contato foi realizado com a direção das escolas participantes, visando, nesse momento, identificar os alunos e famílias.

Foi confeccionada uma carta-convite para os pais de todos os alunos sorteados informando sobre o estudo e sobre o fato de que um membro da equipe do estudo entraria em contato por telefone ou diretamente na residência, para convidá-los pessoalmente para a participação no estudo. Essa carta-convite foi enviada pelas professoras das crianças.

Após o envio das cartas, o contato com os responsáveis iniciou-se a partir de visitas domiciliares e mais uma vez os objetivos do estudo foram explicados e o convite realizado. Para aqueles que aceitaram participar, um encontro foi agendado, no qual solicitou-se a assinatura 
do Termo de Consentimento Livre e Esclarecido. Feito isso, os instrumentos de coleta de dados eram aplicados.

No que se refere à coleta de dados, houveram até três tentativas de contato em dias, horários e de formas diferentes com os participantes que não eram encontrados. Àqueles que não aceitaram participar ou com os quais o contato não foi possível nas três tentativas eram substituídos por outros que compunham a amostra de substituição, composta para esse fim e, então, o mesmo procedimento era realizado, ou seja, três tentativas de contato. Quando o convite para a participação na pesquisa era aceito, assinava-se o TCLE e os instrumentos eram, então, apresentados sob a forma de entrevista.

Observa-se que, a fim de manter o rigor do trabalho e não o prejudicar metodologicamente, os participantes de duas escolas foram excluídos da amostra, em razão do número de entrevistas realizadas ter sido inferior a 50\% do previsto. Assim, o nível de confiabilidade da amostra, de $5 \%$ passou a ser de 7\%, totalizando 321 participantes. Dessa forma, cinco escolas participaram do presente estudo, sendo que os resultados são referentes às crianças e responsáveis advindos destas.

Os dados coletados foram todos de natureza quantitativa, sendo que os referentes aos instrumentos padronizados utilizados: SDQ e IEP foram tratados a partir das planilhas de cálculo de cada um deles. Para as análises estatísticas de correlação e de comparação entre grupos, foram utilizados testes não paramétricos, considerando que, embora a amostra tenha sido composta de forma probabilística, as variáveis estudadas não apresentaram uma distribuição normal na população estudada, o que impediu a realização de testes paramétricos.

Dessa forma, para as análises de correlação, foi utilizado o teste de Spearman. Para as análises de comparação entre grupos foi utilizado o teste de Mann-Whitney. Observa-se que os testes foram considerados com resultado significante quando o valor da estatística que o descreve (p-valor) era menor do que 0,05, admitindo-se como probabilidade de erro o valor de 5\%. Para a realização das análises estatísticas, utilizou-se o Software Statística.

\section{RESULTADOS}

Apresentam-se na Tabela 1 os resultados da prevalência de problemas de saúde mental infantil, advindos da aplicação do SDQ.

Observa-se na Tabela 1 que 43\% das crianças participantes, segundo a percepção de seus responsáveis, apresentam escore "Clínico" para a saúde mental, 14\% apresentam escore "Limítrofe" e 43\% não apresentam problemas de saúde mental.

\section{As práticas e estilos parentais e variáveis do contexto familiar}

Na Tabela 2 encontram-se os resultados obtidos com a aplicação do Inventário de Estilos Parentais.

Considerando que a pontuação de cada prática varia de 0 a 12, observa-se que as médias relativas às práticas positivas (Monitoria Positiva e Comportamento Moral) são altas em detrimento das negativas vistas individualmente. No entanto, ao observar a média do IEP, verifica-se um valor baixo, embora não negativo.

Para além dos dados descritos na Tabela 2, considerando os valores de referência do instrumento, o IEP indica o estilo parental adotado pelos responsáveis e, no presente observou-se a predominância dos estilos parentais negativos.

Apresenta-se a Tabela 3 com os resultados referentes às práticas de cuidado dos responsáveis com as crianças.

Como mostram os dados da Tabela 3, metade dos responsáveis relatou que costuma dar "bronca" na criança mesmo sem a existência de um motivo. A grande maioria dos responsáveis (93\%) relatou que possui o hábito de fazer carinho nas crianças e $75 \%$ brincam com as mesmas. No que se refere ao diálogo/conversa estabelecida entre responsável e criança, verifica-se que a maioria dos responsáveis relata que conversa, sendo o assunto principal a escola. Verifica-se também que $45 \%$ das crianças participantes não possuem uma rotina de atividades e horários quando está em casa e que em 43\% das famílias não existem regras e responsabilidades que todos conhecem e cumprem. 
Tabela 1. Prevalência de Problemas de Saúde Mental Infantil Identificados pelo SDQ junto a escolares do ensino fundamental de São Carlos/SP, 2011.

\begin{tabular}{|c|c|c|c|c|c|c|c|c|}
\hline \multirow{3}{*}{ Subescalas } & \multicolumn{6}{|c|}{ Classificação do SDQ } & \multirow{2}{*}{\multicolumn{2}{|c|}{ Total }} \\
\hline & \multicolumn{2}{|c|}{ Saudável } & \multicolumn{2}{|c|}{ Limítrofe } & \multicolumn{2}{|c|}{ Clínico } & & \\
\hline & f & $\%$ & f & $\%$ & f & $\%$ & f & $\%$ \\
\hline Sintomas emocionais & 113 & 35 & 38 & 12 & 170 & 53 & 321 & 100 \\
\hline Problemas de Conduta & 147 & 46 & 48 & 15 & 126 & 39 & 321 & 100 \\
\hline Hiperatividade & 186 & 58 & 33 & 10 & 102 & 32 & 321 & 100 \\
\hline $\begin{array}{l}\text { Problemas de relacionamento } \\
\text { com os colegas }\end{array}$ & 174 & 54 & 46 & 14 & 101 & 31 & 321 & 100 \\
\hline Comportamento pró-social & 293 & 91 & 10 & 3 & 18 & 6 & 321 & 100 \\
\hline Total de dificuldades & 137 & 43 & 45 & 14 & 139 & 43 & 321 & 100 \\
\hline
\end{tabular}

A pontuação para a classificação da escala dos Sintomas Emocionais varia de 0-3 'saudável', 4 'limítrofe' e 5-10 'Clínico'; Problemas de Conduta 0-2 'saudável', 3 'limítrofe' e 4-10 'clínico'; Hiperatividade 0-5 'saudável', 6 'limítrofe' e 7-10 'clínico'; Problemas com Colegas, 0-2 'saudável', 3 'limítrofe' e 4-10 'clínico'; Comportamento Pró-social, 6-10 'saudável', 5 'limítrofe' e 0-4 'clínico'; Pontuação total das dificuldades 0-13 'saudável', 14-16 'limítrofe' e 17-40 'Clínico'.

Tabela 2. Resultados Descritivos do Inventário de Estilos Parentais avaliado junto aos responsáveis por estudantes do Ensino Fundamental de São Carlos/SP, 2011.

\begin{tabular}{lccc}
\hline Práticas Parentais & Média & Desvio Padrão & Coeficiente de Variação \\
\hline Monitoria positiva & 10,47 & 1,81 & 0,17 \\
Comportamento Moral & 10,38 & 1,93 & 5,38 \\
Punição Inconsistente & 3,26 & 2,31 & 1,41 \\
Negligência & 2,39 & 2,11 & 1,13 \\
Disciplina Relaxada & 3,88 & 2,69 & 1,45 \\
Monitoria Negativa & 6,53 & 2,15 & 3,03 \\
Abuso Físico & 2,41 & 2,11 & 1,14 \\
\hline IEP & $\mathbf{2 , 3 7}$ & $\mathbf{8 , 3 5}$ & $\mathbf{0 , 2 8}$ \\
\hline
\end{tabular}

A seguir, apresenta-se a Tabela 4, com os resultados da correlação entre saúde mental da criança e as práticas parentais dos responsáveis.

Os dados apresentados na Tabela 4 evidenciam que quanto mais os responsáveis se utilizam das práticas parentais: punição inconsistente, disciplina relaxada e abuso físico, mais as crianças apresentam problemas de conduta e problemas gerais de saúde mental. Além disso, quanto mais negativo o estilo parental adotado pelos responsáveis (representado pelo IEP) mais as crianças apresentam problemas de saúde mental.
Em relação aos dados do SDQ com as variáveis do QAC, apenas uma única diferença significativa foi encontrada, referente à variável "existência de regras e responsabilidades que todos conhecem e cumprem". Sendo que, o grupo que vive em famílias nas quais existem regras e responsabilidades que todos conhecem e precisam cumprir apresentam significativamente mais a habilidade de se comportar de forma pró-social $(M=8,75)$, quando comparado com o grupo de crianças que não tem regras que todos conhecem e devem ser cumpridas $(M=8,4)$. 
Tabela 3. Práticas de Cuidado dos Responsáveis com as Crianças, estudantes do Ensino Fundamental da cidade de São Carlos/SP, 2011.

\begin{tabular}{lcc}
\hline Variável & f & $\%$ \\
\hline $\begin{array}{l}\text { Responsável costuma conversar com a criança sobre } \\
\text { assuntos da escola }\end{array}$ & 311 & 97 \\
\hline Sim & 10 & 3 \\
Não & & 77 \\
\hline $\begin{array}{l}\text { Responsável costuma conversar com a criança sobre } \\
\text { assuntos de rotina }\end{array}$ & 247 & 23 \\
\hline Sim & 74 & \\
\hline
\end{tabular}

Responsável costuma conversar com a criança sobre assuntos do interesse dela

\begin{tabular}{lcc}
\hline Sim & 270 & 84 \\
Não & 55 & 17 \\
\hline \multicolumn{2}{l}{ Responsável brinca com a criança } & \\
\hline Sim & 241 & 75 \\
Não & 80 & 25 \\
\hline
\end{tabular}

Criança tem uma rotina de horários e atividades quando está em casa

\begin{tabular}{lll}
\hline Sim & 177 & 55 \\
Não & 144 & 45 \\
\hline
\end{tabular}

Na família existem regras e responsabilidades que todos conhecem e cumprem

\begin{tabular}{lll}
\hline Sim & 183 & 57 \\
Não & 138 & 43 \\
\hline
\end{tabular}

\section{DISCUSSÃO}

Em relação à prevalência de problemas de saúde mental infantil, obtida pelo presente estudo a partir do total de dificuldades do SDQ, observou-se uma taxa de aproximadamente metade das crianças com escore "clínico" para a saúde mental, indicando que as crianças necessitam de intervenção especializada. Esse resultado aponta um índice de prevalência bastante superior ao que tem sido encontrado em estudos nacionais, os quais têm indicado taxas de prevalência variando de $10 \%$ a $25 \%^{1,20}$.

Este resultado suscita algumas hipóteses, tais como o fato das crianças participantes do presente estudo serem provenientes exclusivamente de famílias residentes em bairros periféricos da cidade, população vinculada às escolas municipais da cidade de São Carlos. Sabe-se que a baixa renda familiar e o pertencimento a regiões menos favorecidas tem sido considerada como fatores de risco para o desenvolvimento de problemas relacionados à saúde mental 20, 21, 22 .

Aponta-se para a necessidade de ações de prevenção e promoção à saúde, intervenções especializadas em saúde, educação, assistência social e, pensando nas políticas de saúde, perpassa da atenção básica à atenção especializada, constituindo-se um problema de Saúde Pública, indo além da especificidade da saúde mental ${ }^{23}$.

Tabela 4. Correlações Significativas entre o IEP e o SDQ. São Carlos/SP, 2011.

\begin{tabular}{|c|c|c|c|c|c|}
\hline Sub-escalas & $\begin{array}{c}\text { Punição } \\
\text { Inconsistente }\end{array}$ & Negligência & $\begin{array}{c}\text { Disciplina } \\
\text { relaxada }\end{array}$ & Abuso Físico & IEP \\
\hline Sintomas emocionais & ns & ns & ns & ns & ns \\
\hline Problemas de conduta & $0,378^{* *}$ & ns & $0,355^{* *}$ & $0,352 * *$ & $-0,536^{* *}$ \\
\hline Hiperatividade & ns & ns & ns & ns & ns \\
\hline Problemas de relacionamento & ns & ns & ns & ns & ns \\
\hline Comportamento pró-social & ns & ns & ns & ns & ns \\
\hline Total de Dificuldades & $0,380^{* *}$ & ns & $0,310^{* *}$ & $0,368 * *$ & $-0,455^{* *}$ \\
\hline Suplemento de Impacto & ns & ns & ns & ns & ns \\
\hline
\end{tabular}

$*=p<0,05 ; * *=p<0,01$;

ns = não significativo em relação ao valor de p e/ou índice de correlação < que 0,3 
Sobre as práticas e estilos parentais, as práticas punição inconsistente, disciplina relaxada e abuso físico, bem como o índice de estilos parentais se correlacionaram com o escore geral do SDQ. Tais relações reforçam achados da literatura da área. ${ }^{9,10,24,25}$

Além disso, no que se refere às práticas e estilos parentais adotados pelos responsáveis participantes do presente estudo, observou-se que a maioria adota estilo parental de risco para o desenvolvimento de comportamentos antissociais nas crianças ou estilo parental não adequado.

Esse achado remete à discussão a respeito da importância das atitudes dos pais para o desenvolvimento e saúde mental das crianças, bem como sobre o que pode estar envolvido na adoção pelos pais e responsáveis de estilos parentais negativos. Rutter, ${ }^{16}$ ao discorrer sobre os fatores de risco ambientais e os possíveis efeitos que eles podem causar para o desenvolvimento socioemocional infantil, cita que características presentes no relacionamento e na interação entre mãe/pai e filho são determinantes para o desenvolvimento e saúde mental das crianças. Quando tais relações são permeadas por atitudes negativas (negligência e violência, por exemplo), a probabilidade da criança apresentar dificuldades na esfera da saúde mental aumenta, independente de suas características genéticas, que poderiam deixá-la mais ou menos vulnerável a tal situação ${ }^{16}$.

Além disso, as atitudes parentais, quando negativas, são caracterizadas como fatores de risco proximal, pois estão presentes na relação vivenciada diretamente pela criança e sofrem influência dos fatores de risco que ele chama de distais e que podem ser exemplificados pela pobreza e por outros aspectos sociais ${ }^{16}$. Nessa direção, Collins et al. ${ }^{24}$ apontam a necessidade de se avaliar variáveis contextuais extra-familiares que podem exercer influência na forma dos pais educarem seus filhos. Assim, pode-se considerar que as práticas e estilos parentais ocorrem por meio de processos proximais entre a criança e seu cuidador e, portanto, são influenciadas pelos aspectos sociais e históricos presentes no contexto sociocultural do indivíduo ${ }^{24}$.
Nessa perspectiva, considerando que: 1. Os participantes do presente estudo são responsáveis por crianças estudantes de escolas que estão localizadas em regiões periféricas da cidade, atendendo à população de seu entorno; 2. 71\% desses participantes declaram renda de até dois salários mínimos, aponta-se que as famílias desses responsáveis e crianças vivenciam situações estressoras suficientes para interferir e prejudicar as práticas e estilos parentais adotados pelos cuidadores que, por sua vez, prejudicam os processos proximais vivenciados pelas crianças, trazendo consequências negativas para sua saúde mental. Assim, as práticas e estilos parentais negativos parecem constituir um risco proximal para a saúde mental das crianças foco do presente estudo, sendo consequências de uma série de fatores de risco distais, configurando, assim, um mecanismo de risco ${ }^{16}$.

Sobre os fatores de proteção foi possível verificar que o fato de as famílias apresentarem em seu contexto regras e responsabilidades que todos os membros compartilham e cumprem, configurou-se como um possível fator de proteção à saúde mental infantil, na medida em que se relacionou com a subescala "Comportamento Pró-Social" do SDQ, apresentando diferenças estatisticamente significativas entre os grupos, sinalizando que as crianças que vivem em famílias nas quais essa característica existe, apresentam mais a habilidade de se comportar de forma pró-social do que as crianças que não vivenciam essa realidade.

Vale observar que o fato de na família existirem regras e responsabilidades, que de certa forma, organizam a rotina dos membros familiares, inclusive das crianças, faz parte do conjunto de ações que caracterizam as práticas parentais positivas $^{18,25}$. Gomide (2006) aponta que a existência de regras e limites na família, sugeridas e acompanhadas pelos responsáveis, bem como a monitoria das atividades de rotina da criança (monitoria positiva) a partir de um contexto de afeto e respeito (comportamento moral) favorecem o desenvolvimento de comportamentos pró-sociais nas crianças, sendo identificada no presente estudo como um potencial fator de proteção à saúde mental infantil ${ }^{18}$. 
Ferreira e Marturano, ${ }^{15}$ comparando crianças com dificuldades de aprendizagem que apresentavam e não apresentavam problemas de comportamento, encontraram que as crianças que não tinham problemas de comportamento viviam em famílias com maior monitoramento do uso do tempo livre, maior planejamento e acompanhamento do cotidiano da criança e maior supervisão de atividades. As autoras apontam que há necessidade de intervenções preventivas junto às crianças com dificuldades de aprendizagem, que incluam o sistema familiar e se direcionem para as tarefas de desenvolvimento e para os mecanismos de proteção e vulnerabilidade característicos da fase escolar, no contexto das condições de vida e de desenvolvimento desses indivíduos ${ }^{15}$.

De acordo com os apontamentos de Ferreira e Marturano, ${ }^{15}$ o achado do presente estudo sinaliza para uma questão importante a ser considerada tanto pelos profissionais de serviços de saúde e educação que atendem crianças com problemas relativos à saúde mental ou não, bem como pelas políticas e intervenções de prevenção e promoção à saúde mental infantil, no sentido de planejar e implementar estratégias que possibilitem às crianças terem rotinas de atividades organizadas e respeitadas nos ambientes em que vivem, em especial, escola e família.

\section{CONCLUSÃO}

A partir do presente estudo conclui-se que práticas e estilos parentais negativos adotados pelos responsáveis no cotidiano com suas crianças constituem-se como importantes fatores de risco para o desenvolvimento de dificuldades relacionadas à saúde mental nas crianças. Além disso, a existência de regras e responsabilidades familiares que todos os membros conhecem e cumprem se configurou enquanto um fator protetivo à saúde mental das crianças, na medida em que se relacionou com a habilidade das mesmas em se comportarem de forma pró-social.

Duas limitações importantes do presente estudo devem ser consideradas. Uma delas se refere à utilização somente da versão para pais e/ou responsáveis do SDQ, ou seja, a saúde mental infantil foi avaliada a partir de uma única ótica. Além disso, não foi possível abranger um número maior de participantes, nas diferentes regiões do município.

A despeito das limitações, o estudo aponta questões relevantes a serem consideradas não só pelas políticas públicas de saúde que tratam da prevenção e tratamento em saúde mental, mas também pelas políticas de educação, assistência social e outras que possam lidar de forma mais efetiva e responsável com essa realidade.

\section{REFERÊNCIAS}

1. Organização Mundial da Saúde. Strengthening mental health promotion. Geneva, World Health Organization (Fact sheet no.220). 2001.

2. Sá DGF, Bordin IAS, Martin D, Paula C S P. Fatores de risco para problemas de saúde mental na infância/adolescência. Psicologia: Teoria e Pesquisa. 2010; 26(4):643-652.

3. Cid MFB, Matsukura TS. Problemas de Saúde mental em escolares e seus responsáveis: um estudo de prevalência. Revista de Terapia Ocupacional da Universidade de São Paulo. 2014; 26(1):1-10.

4. Matsukura TS, Fernandes ADS, CID MFB. Saúde mental infantil em contextos de desvantagem socioeconômica: fatores de risco e proteção. Cadernos de Terapia Ocupacional da UFSCar. 2014; 22(2): 251-262.

5. Amstalden ALF, Hoffmann MCCL, Monteiro TPM. A política de saúde mental infantojuvenil: seus percursos e desafios. In E. Lauridsen - Ribeiro, O. Y. Tanaka (Orgs). Atenção em Saúde Mental para crianças e adolescentes no SUS.. São Paulo. Editora Hucitec. 2010; p. 33-45.

5. Schwengber DDS, Piccinini CA. O impacto da depressão pós-parto para a interação mãe-bebê. Estudos de Psicologia. 2003; 8(3): 403-411.

6. Fleitlich, BW, Goodman, R. Epidemiologia. Revista Brasileira de Psiquiatria. 2000; 22(2): 2-6.

7. Ferrioli SHT, Marturano EM, Puntel LP. Contexto familiar e problemas de saúde mental infantil no Programa Saúde da Família. Revista de Saúde Pública. 2007; 41 (2): 251-259.

8. Paula CS, Miranda CT, Bordin IA. Saúde mental na infância e adolescência: revisão dos estudos epidemiológicos brasileiros. In E. Lauridsen - Ribeiro; O. Y. Tanaka (Organizadores). Atenção em saúde mental para crianças e adolescentes no SUS (pp. 75-92). São Paulo. Editora Hucitec. 2010. 
9. Costa F T, Teixeira MAT, Gomes WB. Responsividade e exigência: duas escalas para avaliar estilos parentais. Psicologia: Reflexão e Crítica. 2000; 13(3): 465-473.

10. Gomide PIC. Estilos parentais e comportamento antissocial. In A. Del Prette, Z. A. P. Del Prette (Editores), Habilidades sociais, desenvolvimento e aprendizagem: Questões conceituais, avaliação e intervenção (pp. 21-60). Campinas, Alínea Editora. 2003.

11. Leme VBR, Del Prette ZAP, Coimbra, S. Práticas Educativas Parentais e Habilidades Sociais de Adolescentes de Diferentes Configurações Familiares. PSICO. 2013; 44(4):560-570.

12. Cecconello AM, Koller SH. Competência social e empatia: um estudo sobre resiliência com crianças em situação de pobreza. Estudos de psicologia (Natal). 2003; 5 (1): p. 71-93.

13. Salvo C G, Silvares E M, Toni PM. Práticas educativas como preditoras de problemas de comportamento e competência social. Psicologia em Estudo. 2005; 22(2):187-196.

14. Yu M, Ziviani J, Baxter J, Haynes M. Time use, parenting practice and conduct problems in four- to five-year-old Australian children. Australian Occupational Therapy Journal. 2010; 57 (5): 284-292.

15. Ferreira MCT, Marturano EM. Ambiente familiar e os problemas de comportamento apresentados por crianças com baixo desempenho escolar. Psicologia: Reflexão e Crítica. 2002; 15 (1): 35-44.

16. Rutter M. Environmentally mediated risks for psychopathology: research strategies and findings. Journal American Academic Child Adolescent Psychiatry. 2005; 44(1): 3-18.

17. Goodman R. Psychometric properties of the strengths and difficulties questionnaire (SDQ). Journal of the American Academy of Child \& Adolescent Psychiatry. 2001; 40(11): 1337-1345.

18. Cury CR, Golfeto JH. Strengths and difficulties questionnaire (SDQ): A study of school children in Ribeirão Preto. Revista Brasileira de Psiquiatria. 2003; 25(3): 139-145.

19. Gomide PIC. Inventário de Estilos Parentais (IEP): modelo teórico - Manual de aplicação, apuração e interpretação. Petrópolis. Editora Vozes. 2006.

20. Assis SG, Avanci J Q, Oliveira RV. Desigualdades socioeconômicas e saúde mental infantil. Revista de Saúde Pública. 2009, 43 (1): 92-100.

21. Fleitlich BW, Goodman R. Social factors associated with child mental health problems in Brazil: Cross sectional survey. British Medical Journal. 2001. 323: 599-600.

22. Paula CS, Vedovato MS, Bordin I, Barros MGSM, D’Antino MEF, Mercadante M. Saúde mental e violência entre estudantes da sexta série de um município paulista. Revista de Saúde Pública. 200842 (3): 524-528.

23. Cid MFB. Saúde mental de escolares: um estudo de prevalencia e de fatores de risco e proteção. Tese de Doutorado. Programa de Pós-Graduação em Educação Especial. Universidade Federal de São Carlos. São Carlos, SP. 2011.

24. Collins WA, Maccoby EE, Steinberg L, Hetherington EM,Bornstein MH. Contemporary research on parenting: The case gor nature and nurture. American Psychologist. 2000; 55 (2): 218-232.

25. Teixeira MAP, Bardagi MP, Gomes WB. Refinamento de um instrumento para avaliar responsividade e exigência parental percebidas na adolescência. Avaliação Psicológica. 2004; 3 (1): 1-12.

26. Dishion TJ, Mcmahon RJ. Parental monitoring and the prevention of child and adolescent problem behavior: A conceptual and empirical formulation. Clinical Child and Family Psychology Review. 1998; 1(1): 61-75. 